\title{
HyperTwitter: Collaborative Knowledge Engineering via Twitter Messages
}

\author{
Martin Hepp \\ Universität der Bundeswehr München, E-Business \& Web Science Research Group \\ Werner-Heisenberg-Weg 39, 85577 Neubiberg, Germany \\ mhepp@computer.org
}

\begin{abstract}
A recent trend in the evolution of the Web is the massive contribution of small chunks of content by regular users, typically in combination with mechanisms for fostering social interaction. Such is often referred to as microblogging, with Twitter, Identi.ca, and Google Buzz being the most widely known services. In this paper, we propose to use the underlying interaction pattern, and existing respective services, for the collaborative construction and maintenance of structured knowledge representations. We define (1) a syntax for embedding triple-like statements in Twitter messages, (2) develop a transformation into RDF, (3) suggest mechanisms for controlling the inclusion of such statements made by other users, (4) exploit the resulting graph for query expansion, and thereby provide a direct incentive for users to adopt our syntax; and (5) demonstrate the approach by means of a prototype implementation ${ }^{1}$. The resulting RDF graphs can be combined easily with other Semantic Web data.
\end{abstract}

Keywords: Microblogging, Twitter, Extreme Tagging, RDF, Folksonomies, Tagging, Knowledge Acquisition

\section{Overview}

Microblogging services, in particular Twitter, Identi.ca, and Google Buzz have gained wide popularity. A 2009 Nielsen study [1], for example, reports an increase in Twitter user numbers from $475 \mathrm{k}$ to over 7 Million between February 2008 and February 2009. In such services, contributions are limited to very short, plain text messages (140 characters in the case of Twitter), which typically forces users to take some cognitive effort for verbalizing thoughts or at least shortening existing pieces of content prior to publication. Mechanic copy-and-paste will usually fail due to the limited message length, or will at least require a very well-thought selection of the source fragment for the copy-and-paste operation. Thus, the majority of Twitter posts (called "tweets" or "status updates" in jargon) represent some human effort in processing information. Even the relaying of another user's message (called "retweeting") is based on human judgment and reflects a cognitive effort.

Twitter and most other microblogging services support users in filtering relevant content by a simple yet effective syntactical convention for user identifiers

\footnotetext{
${ }^{1}$ For additional details, please see the HyperTwitter Technical Report [26]
} 
(@username) and keywords (\#keyword, called "hashtag" in jargon). This allows spotting messages directed to a particular user or containing a particular keyword effectively. For instance, Twitter users can easily introduce multiple users to each other or point users who are monitoring a particular hashtag to a new Web resource:

epaulsmith : You should talk to apetermiller

\#html5 developers: look at http://foo.com/

Based on simple string comparison techniques for such significant tokens, the service can link the millions of isolated short messages and build a densely meshed graph, representing social proximity and shared interests.

Unfortunately, Twitter hashtags and, to a lesser degree, Twitter user identifiers suffer from tag ambiguity (the same tag may stand for multiple meanings), tag heterogeneity (multiple tags are in use for the same meaning), and the lack of relationships between tags (e.g. super/subtag relations). The same problems are known from traditional social tagging systems; for an overview of those problems, see e.g. [7, pp. 74-76]. In Twitter, for example, it is very common that participants of an academic conference cannot immediately agree upon one authoritative hashtag for that event, which leads to disconnected messages about the same conference, because some posts contain the hashtag \#ekaw10 and others contain \#ekaw2 010.

The user community has only weak social instruments or techniques at hand for dealing with such synonymous tags. Very frequently, Twitter users spotting the use of synonymous tags will post messages like:

Please use \#ekaw instead of \#ekaw10 or \#ekaw2010

Such messages will be visible for any user watching any of the three variants and hopefully foster convergence. Also, we can often see that organizers of events try to stimulate consensus ex ante by publishing a hashtag recommendation.

In this paper, we will describe how a minimal extension of the existing Twitter syntax will allow Twitter users to

1. consolidate multiple synonymous hashtags for their future queries,

2. express hierarchical or other types of relationships between multiple tags,

3. introduce tags for types of properties betweet arbitrary resources, and

4. use popular Web vocabularies like FOAF, SIOC, Dublin Core, GoodRelations, and others inside Twitter messages.

The guiding principle is to provide a mechanism that is (1) immediately useful for the user contributing the additional content but is at the same time (2) suitable for sharing contributions along social networks, so that many people can benefit from it.

From such augmented tweets, we can easily construct an RDF graph that can be used to improve the recall of search operations on Twitter and that can be exported and combined with any other RDF data on the Web of Linked Data. Since all augmented statements remain regular Twitter messages, they can be shared with others via Twitter lists (grouping posts by a selected set of people) or retweeting, i.e. confirming and relaying a message to all individuals reading your own posts. The main idea is to provide a direct incentive for users to contribute useful statements in the extended syntax, which can be shared and used for weaving a Web of Linked Data. 


\section{Collaborative Knowledge Engineering via Twitter Messages}

In this section, we describe how a lightweight syntactical convention can support users of the Twitter microblogging service to (1) consolidate synonymous hashtags relevant to them and (2) author rich contributions for the Web of Linked Data.

\subsection{Motivating Example}

Very often, Twitter users cannot immediately agree upon a single authoritative hashtag for a topic, which makes it hard to spot all tweets related to that topic. Also, individuals and organizations often use multiple Twitter user IDs, which makes it hard to monitor all tweets from these accounts in one turn. Imagine the hashtags \#munich and \#muenchen were in use for the German city of Munich, and the users @mfhepp and @hypertw would relate to the same individual. While we could manually expand a query "\#munich @mfhepp" to "\#munich OR \#muenchen @mfhepp OR @hypertw", we cannot model and thus reuse and share the underlying equivalency relationship. Also, we cannot express more subtle relationships between tags, like the fact that one tag is more specific than another tag, nor model useful relationships between other resources.

\subsection{HyperTwitter Syntax Proposal}

With a lightweight syntactical convention based on the established Twitter syntax for tags ("\#paris") and users (“@mfhepp"), we can empower Twitter users to embed machine-accessible statements into their tweets, which can then be used for query expansion or combined with other RDF data sources. Basically, we (1) suggest to use "=" or "sameas" for expressing equivalence between tags or between user IDs, (2) "subtag" for expressing that one tag is more specific than a second one, (3) allow introducing arbitrary new properties between elements by means of a preceding greater sign, and (4) support popular CURIEs [2] (e.g. foaf : knows).

Our proposed syntax for triple-like statements inside Twitter messages ("trippletweets") is as follows:

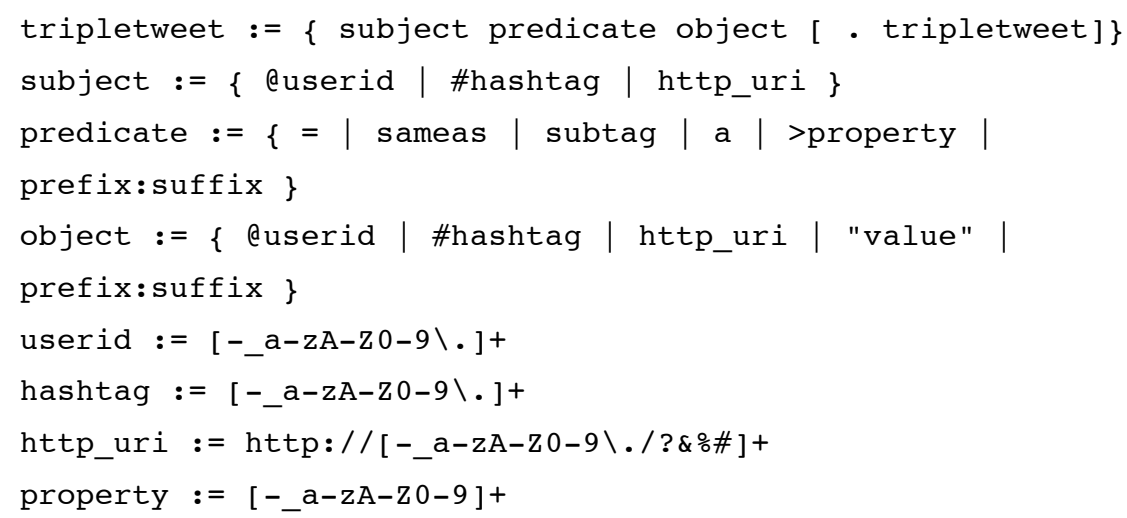




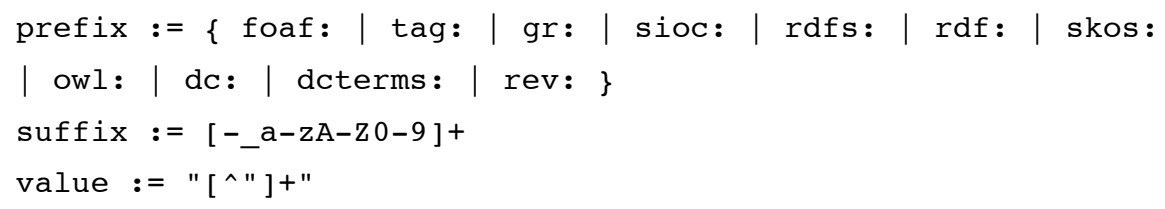

The elements subject, predicate, and object, as well as multiple tripletweets must be separated by one or more valid whitespace characters in the given encoding. The combination of prefix:suffix is a subset of all CURIEs [2].

\subsection{Usage}

In the following, we illustrate the use of our proposed syntax.

\section{Simple examples}

\#newyork sameas \#nyc

The hashtag \#newyork is equivalent to \#nyc (formally: tag:equivalentTag).

\#iswc09 subtag \#iswc

\#tennis subtag \#sports

The hashtag \#iswc09 is a specialization of \#iswc and the hashtag \#tennis is a specialization of \#sports.

@mfhepp = @martinhepp

The user@mfhepp is the same individual as the user@martinhepp. Note that the formal semantics of "=" and "sameas" depends on the type of the subject and object of the statement. For pairs of tags, it is tag:equivalentTag, for individuals it is owl : sameAs, and for http_uris is also owl:sameAs. For other pairs of entities, the statement is unsupported.

\section{Using predefined vocabularies}

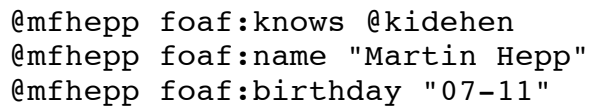

The user@mfhepp knows the user@kidehen. The name of user@mfhepp is “Martin Hepp. His birthday is July 11.

\#iswc09 skos:broader \#iswc

The tag \#iswc09 is related to the tag \#iswc via the skos:broader property. This is equivalent to "\#iswc09 subtag \#iswc".

amicrosoft a gr:BusinessEntity

@microsoft rdf:type gr:BusinessEntity

The user@microsoft is an instance of the class BusinessEntity in the GoodRelations ontology. 


\section{Introducing new tags for types of relationships}

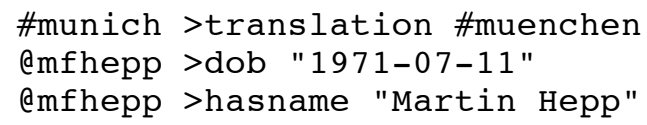

The hashtag \#munich is related to the hashtag \#muenchen via a property labeled with "translation". The user @mfhepp has a property labeled "dob" (date of birth) with the value "1971-07-11".

\section{Using http URIs}

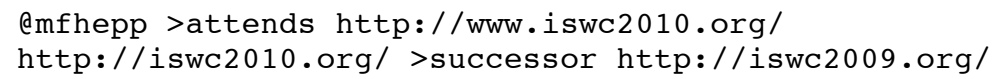

The user@mfhepp is related via a relationship labeled "attend" to something for which the Web page is http://www.iswc2010.org/. Note that the Web of Linked Data requires distinct URIs for events and for Web pages about events, so we cannot use the HTTP URI directly as the object of the statement but have to mint a new URI and link back to the original URI via foaf:topic. Also note that abbreviated URIs (bit.ly etc.) should be expanded prior to that, but are not in the current prototype.

\section{Multiple statements in a single tweet}

@mfhepp foaf:knows @kidehen. @mfhepp foaf:name "Martin Hepp"

The user@mfhepp knows the user@kidehen and the real name of @mfhepp is "Martin Hepp". Note that the whitespace is significant in here.

\subsection{Representation in RDF}

In the following, we describe how rich statements matching our syntax can be represented in RDF. This allows both the flexible implementation of query expansion (e.g. whether you just want to expand hashtags and user IDs by equivalent ones, or expand a query from a single user to everybody from his social network), and other more generic usages of the data.

As a key design choice, we suggest one global namespace for all hashtags in Twitter. This increases access to tags but excludes tag disambiguation. Our motivation for that decision is that in Twitter, other than in typical tagging systems, tags are first and foremost used as tokens to receive the attention of others, i.e., they are designed to be global. Of course, multiple users or communities may produce "tag collisions" by that, but there is a strong incentive to use globally valid hashtags. In Twitter, hashtags have a stronger global reach than in other social tagging systems, because one does not invent tags for personal retrieval but for being visible by others.

Note that this does not mean than one could not control the subset of statements to be used for personal query expansion or other purposes, because HyperTwitter allows defining the single user or group of users whose messages should be parsed and included in the RDF representation. See subsection 2.5 for more details.

Our RDF transformation uses the three Web ontologies FOAF, SKOS, and the Tag Ontology for representing users, tags, and popular relationships. 
Note that in the following, the prefixes definitions for the URIs of HyperTwitter users, tags, properties, and data elements are omitted for brevity. They can be found at http://semantictwitter.appspot.com/.

\section{Users}

users:<userid> a foaf:Agent;

Note: The preceding hash is not included in the URI.

Tags

tags: <hashtag> a tag:Tag;

tag: name "<hashtag>".

\section{HTTP URIs}

data: <http_uri> foaf:topic <http_uri>.

All non-standard characters of the string $<$ http_uri $>$ will be encoded before compiling the subject URI. For example, the URI http://purl.org/ will be represented as

data: http $\% 3 \mathrm{~A} \% 2 \mathrm{~F} \% 2 \mathrm{Fpurl}$. org $\% 2 \mathrm{~F}$

It may be a good idea to first try to expand abbreviated URIs (e.g. http://bit.ly/...).

\section{Property tags}

props:<property> a rdf:Property .

New properties do not immediately have any formal semantics beyond being rdf:Property, but can be found via SPARQL queries. Then, heuristics can be applied based on the frequency of usage, the types of values attached, and the lexical analysis of the property name.

\section{CURIEs for Properties}

For the supported prefixes, i.e. foaf, tag, gr, sioc, rdfs, rdf, owl, skos, dc, dcterms, and rev, the standard expansion to full URIs as per [2] is being used.

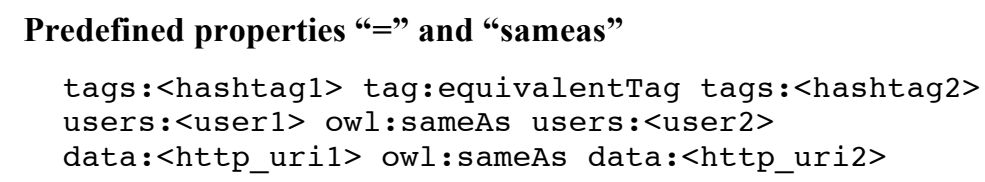

The formal semantics of "=" / "sameas" depends on the type of the subject and object of the statement. For pairs of tags, it is tag: equivalentTag, for individuals it is owl:sameAs, and for http_uris it is also owl:sameAs. For pairs of other types, such statements are ignored in the transformation.

\section{Predefined property "subtag"}

tags: <hashtag1> skos:broader tags: <hashtag2>

We use the SKOS [3] properties for hierarchy relations. Note that this is supported for pairs of tags only. 


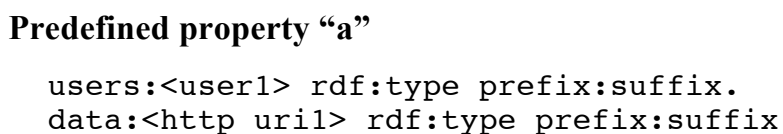

The predefined property "a" is equivalent to $r d f$ :type and can be meaningfully applied only to users and newly minted http URIs as the subject, and classes in the supported Web vocabularies, given as CURIEs, as the object.

\subsection{Trust and Filtering}

It is important to be able to control the origin of tweets to be included. For example, different users or group of users may disagree on whether particular hashtags are equivalent for them. We propose to use existing Twitter techniques for selecting subsets of tweets for a particular purpose: In the simplest form, a user will trust only his / her own tweets containing statements of equivalence or hierarchical relationships for query expansion. Alternatively, one can manage a specific list on Twitter that defines a set of users whose tweets should be considered for query expansion or other purposes. Such lists can be private or public.

Even if one decides to trust one's own statements only, it is possible to find and use other users' statements by simply retweeting them. So if a friend of yours makes the statement " $<$ hashtag $1>=<$ hashtag $2>$ " and you find that useful, you can add it to your own query expansion and relay it to people following you in one turn by retweeting.

\section{Implementation and Evaluation}

A reference implementation of the HyperTwitter syntax is available at http://semantictwitter.appspot.com/. The overall goal was to provide a service that is immediately useful for each individual user, thus creating an incentive for adopting the proposed syntax. At the same time, the RDF content of all public Twitter messages is made accessible for further research and novel applications. For more details on the implementation and a preliminary evaluation, please check the HyperTwitter Technical Report [26].

\section{$4 \quad$ Related Work}

HyperTwitter is related to and partly inspired by the following branches of work.

Meta-models of Tagging and Extensions: While tagging was introduced as an informal technique for attaching descriptors to resources in a collaborative setting, mainly to support the performance of retrieval, the huge amount of respective tagging data soon triggered interest in research to exploit this data for deriving more formal knowledge representations; for an overview of related questions, see Gruber [4]. A first step was the development of ontologies for sharing tags and tagging data, with Newman's Tag Ontology [5] being an early approach that has gained wide popularity. Other researchers proposed extended tag ontologies, mainly for facilitating access to the underlying social structures of tagging and for supporting the semantic enrichment 
of tags, e.g. by disambiguating homonymous tags. Two major efforts in this direction are SCOT [6] and MOAT [7]. For a comprehensive review of tag ontologies, see [8] and [9]. Our approach uses Newman's tag ontology for representing the tag entities found in HyperTwitter statements. This could be mapped easily to equivalent classes in additional tag ontologies.

The two most relevant works (and a direct inspiration) for creating the HyperTwitter prototype are Extreme Tagging [10] and Tag4Tags [11]. Both basically suggest to expand the domain of tagging activities from tagging a resource to tagging tags (in the case of Extreme Tagging), and other types of resources. Such can help to use tagging for consolidating personal or public tag usage and for authoring knowledge representations, since triples of tags can be understood as triples in the RDF model. HyperTwitter applies the idea of Tags4Tags and Extreme Tagging to the significant tokens in free-text microblogging, e.g. user IDs and hashtags.

Syntactical Conventions for Embedding Semantics into Microblogging: Almost all microblogging services rely on simple syntactical conventions for marking up content in messages, e.g. using the hash sign as a prefix for tags/keywords and the "at" sign for user IDs. In the past three years, several proposals have been made to define additional syntactic conventions for representing richer structures.

On the high end of granularity is MicroTurtle ( $\mu \mathrm{ttl})$ [12] by Inkster, a specification for embedding small RDF graphs into microblogging messages using the Turtle syntax. MicroTurtle is very similar to our approach. The main differences are that (1) we use a simpler, linear syntax that is closer to tagging than to RDF and has convenient shortcuts for tag consolidation, which is likely a key motivation for users to use rich structures in messages; (2) we did develop and deploy a reference implementation, and (3) we introduced a simple yet effective message for managing the inclusion of messages to the semantic representation by "trust" lists or user IDs.

TwitterData [13] is a proposal by Fast and Kopsa for encoding property-value pairs in Twitter messages, e.g. "San Francisco Airport \$lat 37.612804 \$long -122.381687". Other than HyperTwitter, it focuses on property-value pairs instead of triples. Also, a mapping to Semantic Web standards has been announced but is not yet available. MicroSyntax [14] is a community effort to identify and document lightweight syntactical conventions for encoding information in Twitter messages and other short user contributions. The initiative also aims at supporting convergence among competing syntaxes and at creating reference implementations. Picoformats [15] is an initiative led by Messina to define syntactical conventions for communications and for executing simple commands via short text messages, originally intended for command-line interfaces, SMS, and other devices. It also refers to other conventions, e.g. MicroTurtle or TwitterData. Twitter Nanoformats [16] is another specification for embedding lightweight semantics into short messages. For example, it suggests the prefixes "L:" for locations, "event:" for events, and "time:" for temporal data. Triple tags, also called "machine tags" on Flickr and on other services, are a convention for representing property-value pairs with explicit namespacing in short messages [17]. A popular usage is geo data, e.g. "geo:long=50.123456". 
Semantic Microblogging: Very recently, there have been several proposals of lifting Twitter content to the Semantic Web technology stack in order to make it accessible for SPARQL queries that combine Twitter data with other RDF data on the Web. For example, SemanticTweet by Flinter is a straightforward service that automatically constructs a FOAF graph from a Twitter user's social network [18].

More sophisticated contributions are on one hand the work by Nowack [19] and on the other hand the SMOB (Semantic-MicrOBlogging) framework [20]. Nowack's work lifts Twitter content to RDF and makes it accessible to SPARQL. While the usage of machine tags (see above) is being discussed, it does not support the authoring of explicit triple statements in tweets, limiting the accessible content to user IDs, SIOC relations, URIs, and property-value pairs. SMOB allows exposing Twitter content in RDF in a similar way but also supports aligning tags or modeling relationships using existing vocabularies. The focus in SMOB is on exposing the obvious meta-data using standard vocabularies. A main difference of our approach is that we additionally foster the introduction of new tags for relationships so that the convenience of free tagging can be used for predicates as well.

Other works: Approaches of maintaining and consolidating tags have been discussed by several authors, e.g. by Golov, Weller, and Peters [21]. Their TagCare system allows users to collate their tags from multiple tagging systems and to express semantic relations for future query expansion and other purposes.

Another stream of research aims at mining ontologies from tagging data, which can also be used for query expansion. A prominent example is the work by Specia and Motta [22]. They propose an automatic approach for deriving formal relations between tags from the combination of tagging data, existing Web ontologies, and other Web resources like Wikipedia. A major difference to our work is that the focus is on a fully automated extraction of formal representation, while we provide a syntax and application for the contribution of human judgment.

As far as Twitter query expansion is concerned, there are already first approaches, e.g. TipTop [23], a semantic Twitter-based search engine which seems to use mining and NLP techniques to extract relevant content for a given search from Twitter. However, it does not provide any RDF export of the data and can thus not be integrated with other Semantic Web resources or technology.

\section{Discussion and Conclusion}

At the time of writing, the amount of Twitter messages posted reaches 50 million tweets per day, which is an average of 600 tweets per second [24]. That means that users contribute an unprecedented amount of content, time, and intelligence, which may be very rewarding to tap for weaving a dense and current Web of Linked Data. Both for maintaining ontologies and facts in knowledge bases, the delayed inclusion of user feedback has kept on being a major bottleneck towards powerful intelligent knowledge-based systems; for a discussion, see e.g. [25]. Our approach reuses the ideas of Extreme Tagging [10] and Tags4Tags [11], i.e. using free tagging for modeling new types of relationships, for the challenge of knowledge authoring by means of microblogging. 
Acknowledgments: HyperTwitter is inspired by the Tags4Tags approach as described by Leyla Jael García-Castro et al. [11].

\section{References}

1. NielsenWire: Twitter's Tweet Smell Of Success, available at http://blog.nielsen.com/nielsenwire/online mobile/twitters-tweet-smell-of-success/.

2. CURIE Syntax 1.0. A syntax for expressing Compact URIs. W3C Candidate Recommendation 16 January 2009, available at http://www.w3.org/TR/curie.

3. SKOS Simple Knowledge Organisation System Reference. W3C Recommendation 18 August 2009, available at http://www.w3.org/TR/skos-reference.

4. Gruber, T.R.: Ontology of Folksonomy: A Mash-Up of Apples and Oranges. International Journal on Semantic Web and Information Systems (IJSWIS) 3 (2007) 1-11

5. Tag Ontology: An Ontology for Tags, available at http://www.holygoat.co.uk/owl/redwood/0.1/tags/.

6. SCOT: Let's Share Tags!, available at http://scot-project.org/.

7. Passant, A., Laublet, P., Breslin, J.G., Decker, S.: A URI is Worth a Thousand Tags: From Tagging to Linked Data with MOAT. International Journal on Semantic Web and Information Systems (IJSWIS) 5 (2009) 72-94

8. Kim, H.L., Passant, A., Breslin, J.G., Scerri, S., Decker, S.: Review and Alignment of Tag Ontologies for Semantically-Linked Data in Collaborative Tagging Spaces. 2008 IEEE International Conference on Semantic Computing (ICSC2008). IEEE Computer Society Washington, DC, USA, Santa Clara, CA, USA (2008) 315-322

9. Kim, H.L., Scerri, S., Breslin, J.G., Decker, S., Kim, H.-G.: The State of the Art in Tag Ontologies: A Semantic Model for Tagging and Folksonomies. International Conference on Dublin Core and Metadata Applications, Berlin, Germany (2008) 128-137

10. Tanasescu, V., Streibel, O.: Extreme Tagging: Emergent Semantics through the Tagging of Tags. First International Workshop on Emergent Semantics and Ontology Evolution (ESOE 2007), Vol. 292. CEUR-WS.org, Busan, Korea (2007) 84-94

11. García-Castro, L., Hepp, M., García, A.: Tags4Tags: Using Tagging to Consolidate Tags. 20th International Conference on Database and Expert Systems Applications (DEXA 2009), Vol. LNCS 5690. Springer, Linz, Austria (2009) 619-628

12. MicroTurtle ( $\mu \mathrm{ttl})$, available at http://buzzword.org.uk/2009/microturtle/spec.

13. Twitter Data. A Simple, Open Proposal for Embedding Data in Twitter Messages, available at http://twitterdata.org/.

14. Microsyntax.org: Deep Structure of the Real-time Stream, available at http://www.microsyntax.org/about-microsyntax/.

15. Picoformats, available at http://microformats.org/wiki/picoformats.

16. Microblogging Nanoformats: Twitter (or Jaiku) Nanoformats Proposal, available at http://microformats.org/wiki/microblogging-nanoformats.

17. Tag (metadata), section: Triple Tags, available at http://en.wikipedia.org/wiki/Triple tag\#Triple tags.

18. SemanticTweet. Twitter Meets the Semantic Web, available at http://semantictweet.com/.

19. Turn Twitter Into Your Personal Assistant, available at http://www.devx.com/semantic/Article/40869.

20. SMOB: Semantic-MicrOBlogging Framework, available at http://smob.me/.

21. Golov, E., Weller, K., Peters, I.: TagCare: A Personal Portable Tag Repository.: Proceedings of the Poster and Demonstration Session at ISWC2008, Vol. 401. CEUR-WS.org, Karlsruhe (2008)

22. Specia, L., Motta, E.: Integrating Folksonomies with the Semantic Web. In: Franconi, E., Kifer, M., May, W. (eds.): 4th European Semantic Web Conference (ESWC 2007), Vol. LNCS 4519. Springer, Innsbruck, Austria (2007) 624-639

23. TipTop Search Engine, available at http://feeltiptop.com/.

24. Measuring Tweets. Twitter Blog Post, February 22, 2010., available at http://blog.twitter.com/2010/02/measuring-tweets.html.

25. Hepp, M.: Possible Ontologies: How Reality Constrains the Development of Relevant Ontologies. IEEE Internet Computing 11 (2007) 90-96

26. Martin Hepp: HyperTwitter: Collaborative Knowledge Engineering via Twitter Messages, Technical Report 2010-01, Universität der Bundeswehr München. PDF at http://www.heppnetz.de/files/hypertwitter-TR.pdf 